\title{
STUDY ON THE IMPROVEMENT OF PHYSICAL-MECHANICAL CHARACTERISTICS IN THE EVA/PVC BLENDS IRRADIATION WITH ELECTRON BEAM IN THE PRESENCE OF TRIALLYLISOCYANURATE
}

\author{
STUDIU PRIVIND ÎMBUNĂTĂTIREA CARACTERISTICILOR FIZICO-MECANICE ALE AMESTECURILOR \\ EVA/PVC IRADIATE CU ELECTRONI ACCELERATI ÎN PREZENTĂ DE TRIALILIZOCIANURAT
}

\author{
Maria Daniela STELESCU ${ }^{1}$, Dana GURAU ${ }^{1}$, Gabriela CRACIUN $^{2}$, Elena MANAILA ${ }^{2 *}$ \\ ${ }^{1}$ National Research \& Development Institute for Textiles and Leather, Division: Leather and Footwear Research Institute, 93 lon Minulescu St., \\ 031215, Bucharest, Romania
}

${ }^{2}$ National Research \& Development Institute for Laser, Plasma and Radiation Physics, 409 Atomistilor, 077125, Magurele, Romania

\section{STUDY ON THE IMPROVEMENT OF PHYSICAL-MECHANICAL CHARACTERISTICS IN THE EVA/PVC BLENDS IRRADIATION WITH ELECTRON BEAM IN THE} PRESENCE OF TRIALLYLISOCYANURATE

ABSTRACT. The paper presents irradiation of thermoplastic elastomers based on ethylene-vinyl acetate (EVA) and poly(vinyl chloride) (PVC) plasticized with small ionizing radiation doses, in the presence of triallylisocyanurate (TAC), for the purpose of improving their characteristics. Results obtained from the study have shown that by irradiating blends of EVA/PVC in presence of TAC, new materials with improved properties have been obtained, as a result of crosslinking reactions and reorientation of sample particles under the influence of accelerated electrons. The optimal irradiation dose and the optimal TAC concentration can be chosen depending on the composition of each blend and on the applications of the final product.

KEY WORDS: EVA, PVC, TAC, irradiation, physico-mechanical properties

STUDIU PRIVIND ÎMBUNĂTĂJIREA CARACTERISTICILOR FIZICO-MECANICE ALE AMESTECURILOR EVA/PVC IRADIATE CU ELECTRONI ACCELERATI ÎN PREZENTA DE TRIALILIZOCIANURAT

REZUMAT. Lucrarea prezintă iradierea unor elastomeri termoplastici pe bază de etilen vinil acetat (EVA) şi policlorură de vinil (PVC) plastifiată cu doze mici de radiaţie ionizantă, în prezenţă de trialilizocianurat (TAC), cu scopul de a îmbunătăţi caracteristicile acestora. Rezultatele obţinute în urma studiului au arătat că prin iradierea amestecurilor de EVA/PVC în prezenţa TAC, s-au obţinut materiale cu proprietăţi îmbunătăţite, ca urmare a reacţiilor de reticulare şi de reorientare a particulelor probelor sub influenţa electronilor acceleraţi. Doza optimă de iradiere şi concentraţia optimă de TAC se pot alege în funcţie de compoziţia fiecărui amestec în parte şi de utilizările produsului final.

CUVINTE CHEIE: EVA, PVC, TAC, iradiere, proprietăţi fizico-mecanice

ETUDE SUR L'AMÉLIORATION DES CARACTÉRISTIQUES PHYSIQUES ET MÉCANIQUES DES MÉLANGES EVA/PVC IRRADIÉS PAR FAISCEAU D'ÉLECTRONS DANS LA PRÉSENCE DE TRIALLYL ISOCYANURATE

RÉSUMÉ. Cet article présente l'irradiation d'élastomères thermoplastiques mélanges à base d'éthylène-acétate de vinyle (EVA) et le chlorure de polyvinyle (PVC) plastifié avec de faibles doses de rayonnements ionisants en présence du triallyl isocyanurate (TAC) afin d'améliorer leurs propriétés. Les résultats obtenus à partir de l'étude ont montré que par l'irradiation des mélanges EVA/PVC en présence de TAC on a obtenu des matériaux avec des propriétés améliorées en raison des réactions de réticulation et la réorientation des particules d'échantillons sous l'influence des électrons accélérés. La dose optimale de radiation et la concentration optimale de TAC peuvent être choisies selon la composition de chaque mélange séparément et selon les utilisations du produit final.

MOTS CLÉS: EVA, PVC, TAC, irradiation, propriétés physiques et mécaniques

\section{INTRODUCTION}

Blends of ethylene-vinyl acetate (EVA) copolymers and poly(vinyl chloride) (PVC) have been extensively studied by many authors by means of different techniques [1-3]. The great interest for EVA/PVC mixtures is due to the fact that EVA, being a highly flexible polymer, can act as plasticizer of PVC, with the advantage to avoid the addition of low molecular plasticizers. In fact the migration of these

\section{INTRODUCERE}

Amestecurile din copolimeri etilen vinil acetat (EVA) şi policlorură de vinil (PVC) au fost studiate extensiv de mulţi autori, utilizând diverse tehnici [1-3]. Interesul semnificativ pentru amestecurile EVA/PVC se datorează faptului că EVA, un polimer foarte flexibil, se poate comporta ca un plastifiant pentru PVC, având avantajul de a nu necesita adăugarea unor plastifianţi cu moleculă mică. De fapt, migrarea celor din urmă în

\footnotetext{
* Correspondence to: Elena MANAILA, National Research \& Development Institute for Laser, Plasma and Radiation Physics, 409 Atomistilor, 077125, Magurele, Romania, email: elena.manaila@yahoo.com
} 
latter in PVC matrices is a considerable problem, especially as regards the stiffening of the final products as well as the potential health risks for medical and food packaging applications. EVA/PVC is an interesting system also because it is an example of homopolymer-copolymer miscible blend although the homopolymers from the three repeating units are immiscible among themselves: PVC is immiscible with polyethylene and also with poly(vinyl acetate), but forms miscible mixtures with EVA within a specified range of copolymer composition. It has been suggested that the dependence of miscibility on the copolymer composition is due to repulsion forces between the two different copolymeric units [1-5].

The paper presents irradiation of blends based on EVA and PVC with small ionizing radiation doses, in the presence of triallylisocyanurate (TAC), for the purpose of improving their characteristics. Through electron beam (EB) irradiation the purpose is to obtain: (a) optimization of interfacial tension; (b) stabilization of the morphology against high stress during development; and (c) enhanced adhesion between the phases in the solid state [6-8]. Thus, compatibility between the two phases is improved and, as a result, better characteristics are obtained. The main advantages of sample irradiation compared to other methods used in processing elasto-plastic materials to improve their properties are: (a) it can be directly applied to the finished product, offering dimensional stability, (b) due to ionizing radiation properties, certain characteristics can be improved or products with unique properties can be obtained, which cannot be achieved using other methods, (c) by using high power accelerators volatile organic compound emissions are reduced, energy is better used and a more exact control of the process is obtained [8-9]. Research papers have been published suggesting that appropriate polyfunctional monomers (PFMs), also called coagents, in polymer matrix could be used to obtain desired physical properties of the blend at lower irradiation doses. Coagents are multi-functional organic molecules which are highly reactive towards free radicals [10-13]. From previous studies [13-14] it was concluded that the most efficient PFM for EVA copolymer blends has been triallylisocyanurate (TAC). In this paper, the influence of TAC concentration on characteristics of EVA/PVC blends irradiated with EB. matricele de PVC este o problemă importantă, mai ales cu privire la întărirea produselor finale, precum şi riscurile de sănătate ce pot apărea în cadrul utilizărilor în medicină şi la ambalarea produselor alimentare. EVA/PVC este un sistem interesant şi pentru că reprezintă un exemplu de amestec miscibil între un homopolimer şi un copolimer, deşi homopolimerii din cele trei unităţi care se repetă sunt imiscibili între ei: PVC este imiscibil cu polietilena şi cu poliacetatul de vinil, dar formează amestecuri miscibile cu EVA într-un anumit interval al compoziţiei copolimerului. S-a sugerat că dependenţa gradului de miscibilitate de compoziţia copolimerului se datorează forţelor de repulsie dintre cele două unităţi copolimerice diferite [1-5].

Lucrarea prezintă iradierea amestecurilor pe bază de EVA şi PVC cu doze mici de radiaţii ionizante, în prezenţă de trialilizocianurat (TAC), în scopul de a îmbunătăţi caracteristicile acestora. Obiectivul iradierii cu electroni acceleraţi (EA) este de a obţine: (a) optimizarea tensiunii interfaciale; (b) stabilizarea morfologiei împotriva solicitării mari din timpul dezvoltării; şi (c) o adeziune mai bună între faze în stare solidă [6-8]. Astfel, se îmbunătăţeşte compatibilitatea dintre cele două faze şi, prin urmare, se obţin caracteristici mai bune. Principalele avantaje ale iradierii probelor, în comparaţie cu alte metode utilizate la prelucrarea materialelor elasto-plastice pentru a le îmbunătăţi proprietăţile, sunt următoarele: (a) se poate aplica direct pe produsul finit, conferind stabilitate dimensională, (b) datorită proprietăţilor radiaţiei ionizante, se pot îmbunătăţi anumite caracteristici sau se pot obţine produse cu proprietăţi unice, care nu se pot obţine utilizând alte metode, (c) prin utilizarea unor acceleratori de mare putere, se reduc emisiile de compuşi organici volatili, energia este utilizată mai eficient şi se obţine un control mai precis asupra procesului [8-9]. Au fost publicate lucrări de cercetare care sugerează că anumiţi monomeri polifunç̧ionali (PFMs), numiţi şi coagenţi, se pot utiliza în matricea polimerică pentru a obţine proprietăţile fizice dorite pentru amestecuri polimerice la doze mici de iradiere. Coagenţii sunt molecule organice multifuncţionale care au o reactivitate foarte mare faţă de radicalii liberi [10-13]. Din studiile anterioare [13-14] s-a concluzionat că cel mai eficient monomer polifuncţional pentru amestecurile de copolimer EVA a fost trialilizocianuratul (TAC). În această lucrare se va stabili influenţa concentraţiei de TAC asupra caracteristicilor amestecurilor EVA/PVC iradiate cu EB. 


\section{EXPERIMENTAL}

\section{Materials}

The following raw materials were used: (1); ethylene vinyl acetate copolymer Elvax $260 \quad$ (2) plasticized PVC prepared from: PVC with a $64 \mathrm{~K}$-wert value, dioctyl phthalate (DOP), PVC stabilizer (LGP 8008) and antioxidant (Uvinul $5050 \mathrm{H}$ ); (3) polyfunctional monomer triallylcyanurate Luvomaxx TAC DL 70, (4) zinc oxide, (5) stearophanic acid, (6) polyethylene glycol PEG 4000. Table 1 presents the materials used in the mixtures and their main characteristics.

\section{Sample Preparation}

Blends based on EVA and plasticized PVC were obtained in two stages: (1) PVC plasticizing and (2) preparing blends based on EVA and plasticized PVC.

1. PVC plasticizing was accomplished by plasticizer (DOP) absorption into PVC when mixing in a $2 \mathrm{~L}$ vessel of plasticorder PLV 330 Brabender at 70 rpm, temperature of $40^{\circ} \mathrm{C}$ for $10 \mathrm{~min}$. For a good thermal stability, temperature stabilizer and antioxidants have been introduced over time. The resulted plasticized PVC is processed into a sheet on a laboratory roll electrically heated, the resulted sheet being used in the next stage in the blend preparation. Table 2 presents the recipe of PVC plasticizing blend.

2. Blends containing EVA, plasticized PVC, zinc oxide, stearophanic acid and polyethylene glycol were prepared by mixing on an electrically heated laboratory roll at max. $100^{\circ} \mathrm{C}$, working time $5^{\prime}$. Plates of $150 \times 150 \times 2$ $\mathrm{mm}^{3}$ were prepared to determine physico-mechanical properties using an electrical laboratory press. Press working temperature was $160^{\circ} \mathrm{C}$, pre-heating time $2^{\prime}$, modeling time $5^{\prime}$ and room temperature cooling time 2'. EVA/plasticized PVC blends were developed, containing 100, 75, 50 and $25 \%$ (mass percentages) EVA respectively. In the blend containing 25\% EVA and $75 \%$ plasticized PVC, TAC was introduced in amounts of $3 \mathrm{phr}$ (parts to 100 parts elastomer), 6 phr, 9 phr and 12 phr, in order to determine the influence of this type of polyfunctional monomer on the characteristics of such a blend. The TAC polyunctional monomer was selected to be used in crosslinking by electron beam irradiation of EVA as a result of previous studies [14].

\section{PARTEA EXPERIMENTALĂ}

\section{Materiale}

S-au utilizat următoarele materii prime: (1) copolimer etilen vinil acetat Elvax 260; (2) PVC plastifiat preparat din: PVC cu Kw 64, dioctilftalat (DOF), stabilizator pentru PVC (LGP 8008) şi antioxidant (Uvinul 5050H); (3) monomer polifuncţional trialilcianurat Luvomaxx TAC DL 70, (4) oxid de zinc, (5) acid stearic, (6) polietilen glicol PEG 4000. Tabelul 1 prezintă materialele utilizate în amestecuri şi principalele lor caracteristici.

\section{Pregătirea probelor}

Amestecurile pe bază de EVA şi PVC plastifiat au fost obţinute în două etape: (1) plastifierea PVC-ului şi (2) pregătirea amestecurilor pe bază de EVA şi PVC plastifiat.

1. Plastifierea PVC-ului s-a realizat prin absorbţia plastifiantului (DOF) în PVC în timpul amestecării în vasul de 2 L al Plasticorder-ului PLV 330 Brabender la 70 rpm, la temperatura de $40^{\circ} \mathrm{C}$ timp de $10 \mathrm{~min}$. Pentru o stabilitate termică bună în timp, s-au introdus stabilizatorul de temperatură şi antioxidanţi. PVC-ul plastifiat rezultat s-a prelucrat sub formă de foaie pe un valţ de laborator cu încălzire electrică, iar foaia rezultată s-a utilizat în etapa următoare, la pregătirea amestecului. Tabelul 2 prezintă receptura amestecului de PVC plastifiat.

2. Amestecurile care conţin EVA, PVC plastifiat, oxid de zinc, acid stearic şi polietilen glicol s-au realizat prin tehnica amestecării pe un valţ de laborator cu încălzire electrică la max. $100^{\circ} \mathrm{C}$, timp de lucru 5'. S-au realizat plăci de $150 \times 150 \times 2 \mathrm{~mm}^{3}$ pentru determinarea proprietăţilor fizico-mecanice utilizând o presă electrică de laborator. Temperatura de lucru la presă a fost $160^{\circ} \mathrm{C}$, timp de preîncălzire $2^{\prime}$, timp de modelare $5^{\prime}$ şi timp de răcire la temperatura camerei 2 '. S-au realizat amestecuri EVA/PVC plastifiat care conţin 100, 75, 50, 25\% (procente de masă) EVA. În amestecul care conţine 25\% EVA şi 75\% PVC plastifiat s-a introdus TAC în cantităţi de 3 phr (părţi la 100 părţi elastomer), 6 phr, 9 phr şi 12 phr, pentru a se determina influenţa acestui tip de monomer polifuncţional asupra caracteristicilor amestecului. Monomerul polifuncţional TAC a fost selectat pentru a fi utilizat la reticularea prin iradiere $\mathrm{cu}$ electroni acceleraţi a EVA în urma unor studii realizate anterior [14]. 
Table 1: Characteristics of materials used

Tabelul 1: Caracteristicile materialului utilizat

\begin{tabular}{|c|c|c|}
\hline $\begin{array}{l}\text { Materials used } \\
\text { Materiale utilizate }\end{array}$ & $\begin{array}{l}\text { Characteristics } \\
\text { Caracteristici }\end{array}$ & $\begin{array}{l}\text { Chemical structure } \\
\text { Structura chimică }\end{array}$ \\
\hline $\begin{array}{l}\text { Elvax } 260 \\
\text { Ethylene vinyl acetate copolymer } \\
\text { Copolimer etilen vinil acetat }\end{array}$ & $\begin{array}{l}27.8 \% \text { wt\% vinyl acetate content } \\
\text { Flow index (MFI) } 5.5 \mathrm{~g} / 10 \mathrm{~min} \text { at } 190^{\circ} \mathrm{C} \text { and } \\
2.16 \mathrm{~kg} \text { load } \\
\text { Conţinut acetat de vinil: } 27,8 \% \text { wt\% } \\
\text { Indice de curgere: } 5,5 \mathrm{~g} / 10 \mathrm{~min} \text { la } 190^{\circ} \mathrm{C} \text { şi } \\
\text { sarcina de } 2,16 \mathrm{~kg}\end{array}$ & {$\left[\begin{array}{rr}H & H \\
1 & 1 \\
C & -C \\
1 & 1 \\
H & H\end{array}\right]_{n}\left[\begin{array}{rr}\mathrm{H}_{3} \mathrm{C} \\
\mathrm{O}=\mathrm{I} \\
\mathrm{H} & \mathrm{I} \\
1 & 1 \\
\mathrm{C} & -\mathrm{C} \\
1 & 1 \\
\mathrm{H} & \mathrm{H}\end{array}\right]_{\mathrm{m}}$} \\
\hline PVC & $\begin{array}{c}\text { K-wert value } 64 \\
\text { moisture and volatile } 0.2 \% \\
\text { Absorbtion of plasticizer DOP } 100 \% \\
\text { Valoarea K } 64 \\
\text { Umiditate şi materii volatile } 0,2 \% \\
\text { Absorbţia plastifiantului DOP } 100 \%\end{array}$ & 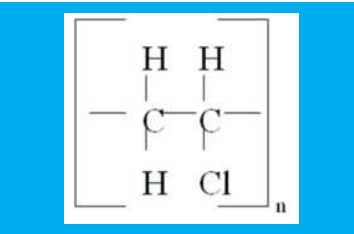 \\
\hline $\begin{array}{l}\text { PVC stabilizer LFR } 8008 \\
\text { Stabilizator PVC LFR } 8008\end{array}$ & $\begin{array}{l}\text { Diatomic lead phosphite content } 10-30 \% \\
\text { Tribasic lead sulphate content } 30-60 \% \\
\text { Lead stearate diatomic content } 10-30 \% \\
\text { Conţinut de fosfit de plumb diatomic } 10-30 \% \\
\text { Conţinut de sulfat de plumb tribazic } 30-60 \% \\
\text { Conţinut de stearat de plumb diatomic } 10-30 \%\end{array}$ & - \\
\hline $\begin{array}{l}\text { Dioctyl phthalate (DOP) } \\
\text { Dioctilftalat (DOF) }\end{array}$ & $\begin{array}{c}\text { Density } 0.984 \mathrm{~g} / \mathrm{cm}^{3} \\
\mathrm{pH} 7 \\
99.5 \% \text { purity } \\
\text { Densitate } 0,984 \mathrm{~g} / \mathrm{cm}^{3} \\
\mathrm{pH} 7 \\
\text { Puritate } 99,5 \%\end{array}$ & $\mathrm{C}_{24} \mathrm{H}_{38} \mathrm{O}_{4}$ \\
\hline $\begin{array}{l}\text { Zinc stearate } \\
\text { Stearat de zinc }\end{array}$ & $\begin{array}{l}\text { Zn content } 11 \% \\
\text { Melting point } 127^{\circ} \mathrm{C} \\
\text { Conţinut } \mathrm{Zn} 11 \% \\
\text { Punct de topire } 127^{\circ} \mathrm{C}\end{array}$ & - \\
\hline Antioxidant Uvinul $5050 \mathrm{H}$ & $\begin{array}{c}\text { Density } 0.99 \mathrm{~g} / \mathrm{cm}^{3} \\
\text { Melting temperature range } 95-125^{\circ} \mathrm{C} \\
\text { Heat stability higher than } 300^{\circ} \mathrm{C} \\
\mathrm{pH} 7 \\
\text { Densitate } 0,99 \mathrm{~g} / \mathrm{cm}^{3} \\
\text { Interval temperatură de topire } 95-125^{\circ} \mathrm{C} \\
\text { Stabilitate termică peste } 300^{\circ} \mathrm{C} \\
\mathrm{pH} 7\end{array}$ & $\begin{array}{l}\text { HALS - Aromatic secondary } \\
\text { amines } \\
\text { HALS - amine secundare } \\
\text { aromatice }\end{array}$ \\
\hline $\begin{array}{l}\text { Polyethylene glycol PEG } 4000 \\
\text { Polietilenglicol PEG } 4000\end{array}$ & $\begin{array}{l}\text { Density: } 1.128 \mathrm{~g} / \mathrm{cm}^{3} \\
\text { Melting point range: } 4-8^{\circ} \mathrm{C} \\
\text { Densitate: } 1,128 \mathrm{~g} / \mathrm{cm}^{3} \\
\text { Interval punct de topire: } 4-8^{\circ} \mathrm{C}\end{array}$ & $\mathrm{H}^{\top}$ \\
\hline $\begin{array}{l}\text { Stearophanic acid } \\
\text { Acid stearic }\end{array}$ & $\begin{array}{l}\text { Titre fatty acids } 59 \\
\text { Mineral acids absence } \\
\text { Titru acizi graşi } 59 \\
\text { Absenţă de acizi minerali }\end{array}$ & - \\
\hline
\end{tabular}


Table 1: Continued

Tabelul 1: Continuare

\begin{tabular}{|c|c|c|}
\hline $\begin{array}{l}\text { Materials used } \\
\text { Materiale utilizate }\end{array}$ & $\begin{array}{l}\text { Characteristics } \\
\text { Caracteristici }\end{array}$ & $\begin{array}{l}\text { Chemical structure } \\
\text { Structura chimică }\end{array}$ \\
\hline $\begin{array}{c}\text { Zinc oxide - } \\
\text { Vulcanization activator } \\
\text { Oxid de zinc-activator de } \\
\text { vulcanizare }\end{array}$ & $\begin{array}{c}\text { Zinc oxide content } 99.2 \% \\
\text { Humidity } 0.15 \% \\
\text { Free zinc } 0.14 \% \\
\text { Conţinut de oxid de zinc } 99,2 \% \\
\text { Umiditate } 0,15 \% \\
\text { Zinc liber } 0,14 \%\end{array}$ & $\mathrm{ZnO}$ \\
\hline $\begin{array}{c}\text { Triallylcyanurate } \\
\text { Luvomaxx TAC DL } 70 \\
\text { Trialilcianurat Luvomaxx TAC DL } 70\end{array}$ & $\begin{array}{l}\text { Melting point: } 26-28^{\circ} \mathrm{C} \\
\text { Boiling point: } 119-120^{\circ} \mathrm{C} \\
26 \% \text { percentage of ash } \\
\text { Density: } 1.34 \mathrm{~g} / \mathrm{cm}^{3} \\
30 \% \text { active synthetic silica } \\
\text { Punct de topire: } 26-28^{\circ} \mathrm{C} \\
\text { Punct de fierbere: } 119-120^{\circ} \mathrm{C} \\
\text { Conţinut cenuşă } 26 \% \\
\text { Densitate: } 1,34 \mathrm{~g} / \mathrm{cm}^{3} \\
\text { Dioxid de siliciu sintetic activ } 30 \%\end{array}$ & \\
\hline
\end{tabular}

Table 2: Recipe of PVC plasticizing blend

Tabelul 2: Receptura de plastifiere a amestecului de PVC

\begin{tabular}{|c|c|}
\hline $\begin{array}{c}\text { Ingredients } \\
\text { Ingrediente }\end{array}$ & $\begin{array}{c}\text { Amounts (g) } \\
\text { Cantităţi (g) }\end{array}$ \\
\hline PVC & 1000 \\
\hline DOP & 500 \\
\hline LFR 8008 & 40 \\
\hline $\begin{array}{c}\text { Zinc stearate } \\
\text { Stearat de zinc }\end{array}$ & 5 \\
\hline Uvinul 5050 & 10 \\
\hline
\end{tabular}

\section{Electron Beam Irradiation}

The samples were packed in a polyethylene film and were irradiated at doses ranging from 5 to $20 \mathrm{Mrad}$ ( $1 \mathrm{Mrad}=10 \mathrm{~Gy}$ ) irradiation in the ILU-6M cavity electron accelerator at room temperature. The accelerator consists mainly of two systems: the electron acceleration system (including resonator, RF generator, vacuum pumps for the accelerating structure etc.) or accelerated electron generator, and accelerated electron scanning system. The ILU-6M is a resonator-type accelerator, operating at $1155 \mathrm{MHz}$. This accelerator generates electron beam pulses of 0.375 ms duration, up to $0.32 \mathrm{~A}$ current peak intensity

\section{Iradierea cu electroni acceleraţi}

Probele au fost învelite în folie de polietilenă şi au fost supuse iradierii la doze în intervalul 5-20 Mrad (1 Mrad = $10 \mathrm{~Gy}$ ) în acceleratorul de electroni ILU-6M la temperatura camerei. Acceleratorul constă în principal din două sisteme: sistemul de accelerare a electronilor (care include rezonatorul, generatorul RF, pompele de vid pentru structura de accelerare etc.) sau generatorul de electroni acceleraţi şi sistemul de scanare cu electroni acceleraţi. Acceleratorul ILU-6M este de tip rezonator şi funcţionează la $1155 \mathrm{MHz}$. Acest accelerator generează impulsuri de electroni acceleraţi de durată $0,375 \mathrm{~ms}$, cu intensitatea maximă a 
and up to $6 \mathrm{~mA}$ mean current intensity. The crosssectional size of the scanned EB at the ILU-6M vacuum window exit is $1100 \mathrm{~mm} \times 65 \mathrm{~mm}$. The EB effects are related to the absorbed dose (D), expressed in Gray or $\mathrm{J} \mathrm{kg}^{-1}$. The single pass dose with conveyor under the ILU-6M scanner is adjustable from $12.5 \mathrm{kGy}$ to $50 \mathrm{kGy}$. The layers of three sandwiched sheets were irradiated by repeatedly passing on a conveyor under the ILU-6M scanner in atmospheric conditions and at room temperature of $25^{\circ} \mathrm{C}$.

According to the Technical Report Series No. 277 [15], the absorbed dose is the major parameter in the accelerated electron radiation. The vulcanizing and grafting process performances are provided by the severe control of this parameter.

The relation defining the absorbed dose is: curentului de până la 0,32 A şi intensitatea medie a curentului de până la $6 \mathrm{~mA}$. Dimensiunea secţiunii transversale a sistemului de scanare a EA la fereastra de ieşire a ILU-6M este $1100 \mathrm{~mm} \times 65 \mathrm{~mm}$. Efectele EA sunt legate de doza absorbită (D), exprimată în Gray sau J kg ${ }^{-1}$. Doza la o singură trecere a transportorului sub scannerul ILU-6M este reglabilă de la 12,5 kGy la 50 kGy. Sandvişurile cu trei foi au fost iradiate prin trecerea repetată pe un transportor sub scanner-ul ILU-6M în condiţii atmosferice şi la temperatura camerei de $25^{\circ} \mathrm{C}$.

Conform Seriei de Rapoarte Tehnice Nr. 277 [15], doza absorbită este principalul parametru în radiaţia cu electroni acceleraţi. Performanţele proceselor de vulcanizare şi grefare sunt date de controlul strict al acestui parametru.

Relaţia care defineşte doza absorbită este:

$$
D=d \varepsilon / d m
$$

where $d \varepsilon$ is the mean energy given up by the ionizing radiation to the mass amounts $\mathrm{dm}$ of the substance interacting with this ionizing radiation. $\mathrm{dm}$ is emphasized to be very low but not so low that the mean energy $d \varepsilon$ given up by the radiation would undergo a significant fluctuation. Absorbed dose is measured in $\mathrm{J} / \mathrm{kg}$. The $\mathrm{SI}$ unit measure for the absorbed dose is the gray (Gy): $1 \mathrm{~Gy}=1 \mathrm{Joule} / \mathrm{kg} ; 10$ $k G y=1$ Mrad.

To control the radiation dose EA, the methodology used was that of measurements of absorbed dose in accelerated electron beams with the chemical system "ceric sulfate - cerous sulfate" according to ISO/DIS 15555/1997 and Manual on Radiation Dosimetry by Niels W. Holm and Roger J. Berry [16].

\section{Laboratory Tests}

Mechanical properties of samples were measured on a Schopper tensile tester with a nominal rate of the traverse of the moving grip of $460 \mathrm{~mm} / \mathrm{min}$. Modulus at $100 \%$ strain, tensile strength and elongation at break tests were carried out according to the conditions described in ISO 37/2012, on dumb-bell shaped specimens of Type 2 . Tearing strength tests were carried out using angular test pieces (type II) according to SR EN 12771/2003. Hardness of materials was measured using the Shore A scale with samples of $6 \mathrm{~mm}$ unde $d \varepsilon$ este energia medie cedată de radiaţia ionizantă cantităţilor de masă, $\mathrm{dm}$, ale substanţei care interacţionează cu această radiaţie ionizantă. Se subliniază că dm trebuie să aibă o valoare mică, însă nu atât de mică încât să ducă la o fluctuaţie semnificativă a energiei medii $d \varepsilon$ cedate de radiaţie. Doza absorbită se măsoară în J/kg. Unitatea de măsură conform SI pentru doza absorbită este Gray (Gy): $1 \mathrm{~Gy}=1 \mathrm{Joule} / \mathrm{kg}$.; 10 kGy $=1 \mathrm{Mrad}$.

Pentru a controla doza de radiaţie cu EA, s-a utilizat metodologia măsurării dozei absorbite în fasciculele de electroni acceleraţi în sistemul chimic "sulfat de ceriu - sulfat ceros" conform ISO/DIS 15555/1997 şi Manualului de dozimetrie a radiaţiilor de Niels W. Holm şi Roger J. Berry [16].

\section{Teste de laborator}

Proprietăţile mecanice ale probelor au fost determinate cu un aparat de încercare mecanică Schopper, la o viteză a capetelor de prindere de 460 $\mathrm{mm} / \mathrm{min}$. Modulul de elasticitate la întindere $100 \%$, rezistenţa la rupere şi alungirea la rupere au fost determinate conform condiţiilor descrise în ISO 37/2012, pe epruvete în formă de halteră de tip 2 . Testarea rezistenţei la sfâşiere s-a efectuat utilizând epruvete de testare unghiulare (de tip II) conform SR EN $12771 / 2003$. Duritatea materialelor s-a măsurat pe scara Shore A pe epruvete de $6 \mathrm{~mm}$ grosime, utilizând 
thickness, by using a hardener tester according to ISO 7619-1/2011. Elasticity was evaluated with a Schoob test machine using $6 \mathrm{~mm}$ thick samples, according to ISO 4662/2009.

\section{RESULTS AND DISCUSSIONS}

\section{Establishing the Composition Action onto the Physico- Mechanical Characteristics}

In order to establish how the compositions acts on the characteristics were prepared blends based on EVA(Elvax 260)/plasticized PVC and containing different percentages: 100, 75, 50, 25 and 0\% (EVA weight from the total polymer mass).

Analyzing the physico-mechanical properties of EVA/plasticized PVC blends in Table 3, it is noticed that the decrease in EVA amount in the blends results in: an irregular variation of hardness and elongation at break, a decrease in elasticity and a minimum value for tear strength and $100 \%$ modulus. These characteristics indicate that EVA/PVC blends have low degrees of miscibility.

Many studies have shown that EVA/PVC blends form one-phase, two-phase and semi-compatible phases. These blends have been thoroughly studied by various techniques [1-6] and it has been shown that by varying the concentration of vinyl acetate (VA) in the copolymer and the composition of EVA in the blend, one may observe the different phase relations. The VA concentration in the EVA copolymer appears to be the controlling factor concerning the compatibility of the EVA/PVC blends. When the VA concentration is less than 40 or greater than 75 weight \%, it is commonly accepted that the corresponding copolymer is immiscible with PVC. The EVA/PVC blend goes through what may be termed a "window of miscibility" when the VA concentration of the copolymer is between 40 and 75 weight \% [3]. In this VA concentration region there exists at least partial compatibility between the EVA copolymer and poly(vinyl chloride). Blends of EVA/PVC containing 60-75 weight \% VA in the EVA copolymer have been found to be completely miscible; whereas, only partial compatibility has been suggested for blends when the VA concentration is between 40 and $60 \%[1-6,17]$. un aparat de testare a durităţii conform ISO 76191/2011. Elasticitatea s-a evaluat cu un aparat Schoob utilizând probe de $6 \mathrm{~mm}$ grosime, conform ISO 4662/2009.

\section{REZULTATE ŞI DISCUTII}

\section{Stabilirea influenţei compoziţiei asupra caracteristicilor fizico-mecanice}

Pentru a stabili influenţa compoziţiilor asupra caracteristicilor, s-au pregătit amestecuri pe bază de EVA (Elvax 260)/PVC plastifiat, cu diferite cantităţi de EVA, şi anume: 100, 75, 50, 25 şi 0\% (procente de masă de EVA din masa totală a polimerului).

Analizând proprietăţile fizico-mecanice ale amestecurilor EVA/PVC plastifiat din Tabelul 3, se observă că, odată cu scăderea cantităţii de EVA din amestecuri, au loc: o variaţie neuniformă a durităţii şi alungirii la rupere, scăderea elasticităţii iar rezistenţa la rupere şi modulul 100\% prezintă un minim. Aceste caracteristici indică faptul că amestecurile EVA/PVC au grad scăzut de miscibilitate.

Multe studii au arătat că amestecurile EVA/PVC formează o fază, două faze şi faze semi-compatibile. Aceste amestecuri au fost studiate în detaliu prin diverse tehnici [1-6] şi s-a demonstrat că variind concentraţia de acetat de vinil (VA) din copolimer şi compoziţie de EVA din amestec, se poate observa diferitele relaţii de fază. Concentraţia de VA din copolimerul EVA pare să fie factorul decisiv în privinţa compatibilităţii amestecurilor EVA/PVC. Când concentraţia de VA este mai mică decât 40 sau mai mare decât 75 procente de masă, copolimerul corespunzător este imiscibil cu PVC. Amestecul EVA/PVC trece prin ceea ce se poate numi "fereastră de miscibilitate" când concentraţia de VA din copolimer se situează între 40 şi 75 procente de masă [3]. În această zonă de concentraţie a VA există compatibilitate cel puţin parţială între copolimerul EVA şi policlorura de vinil. S-a arătat că amestecurile de EVA/PVC care conţin 60-75 procente de masă VA în copolimerul EVA sunt complet miscibile; în timp ce doar o compatibilitate parţială $s$-a sugerat pentru amestecurile cu concentraţie VA între 40 şi 60\% [1-6, 17]. 
Rellick et al. [3] have shown that blends of an ethylene-vinyl acetate copolymer containing $45 \%$ by weight VA (EVA45) and PVC are completely miscible only at the composition extremes of the blend. When the EVA45 concentration is between 10 and $75 \%$, the blends have been described as semi-compatible. There has been some debate regarding the nature of this compatibility and several articles have reviewed this aspect of the EVA45/PVC blend [1-6]. As in the blends we developed EVA concentration is between 10 and $75 \%$, and at the same time the VA concentration is less than 40 weight \%, it is commonly accepted that the corresponding copolymer is immiscible with PVC, therefore EVA/PVC blends obtained have been shown to form two-phase and semi-compatible phases. Physico-mechanical properties of blends obtained confirm these results found in the literature.
Rellick et al. [3] au arătat că amestecurile dintr-un copolimer etilen vinil acetat care conţine 45 procente de masă VA (EVA45) şi PVC sunt complet miscibile doar la valori extreme ale compoziţiei amestecului. Când concentraţia EVA45 se situează între 10 şi 75\%, amestecurile au fost descrise ca fiind semi-compatibile. A existat o dezbatere cu privire la natura acestei compatibilităţi şi câteva articole au analizat acest aspect al amestecului EVA45/PVC [1-6]. Deoarece în amestecurile realizate de noi concentraţia de EVA se situează între 10 şi $75 \%$ şi, în acelaşi timp, concentraţia de VA are mai puţin de 40 procente de masă, copolimerul corespunzător este imiscibil cu PVC-ul, deci s-a demonstrat că amestecurile EVA/PVC obţinute formează două faze şi faze semi-compatibile. Proprietăţile fizicomecanice ale amestecurilor obţinute confirmă aceste rezultate din literatura de specialitate.

Table 3: Physico-mechanical characteristics versus blends composition Tabelul 3: Caracteristicile fizico-mecanice în funcţie de compoziţia amestecurilor

\begin{tabular}{|c|c|c|c|c|c|}
\hline $\begin{array}{l}\text { Characteristics / blends composition } \\
\text { Caracteristici / compoziţia amestecurilor }\end{array}$ & EVA & $\begin{array}{l}\text { EVA } 75 \% \\
\text { PVC } 25 \%\end{array}$ & $\begin{array}{l}\text { EVA } 50 \% \\
\text { PVC } 50 \%\end{array}$ & $\begin{array}{l}\text { EVA } 25 \% \\
\text { PVC } 75 \%\end{array}$ & PVC \\
\hline $\begin{array}{l}\text { Hardness, }{ }^{\circ} \mathrm{ShA} \\
\text { Duritate, }{ }^{\circ} \mathrm{ShA}\end{array}$ & 80 & 81 & 85 & 84 & 87 \\
\hline $\begin{array}{l}\text { Elasticity, \% } \\
\text { Elasticitate, \% }\end{array}$ & 42 & 28 & 18 & 11 & 8 \\
\hline $\begin{array}{c}100 \% \text { Modulus, } \mathrm{N} / \mathrm{mm}^{2} \\
\text { Modul } 100 \%, \mathrm{~N} / \mathrm{mm}^{2}\end{array}$ & 2.8 & 3 & 4.5 & 6.5 & 9.7 \\
\hline $\begin{array}{c}\text { Tensile strength, } \mathrm{N} / \mathrm{mm}^{2} \\
\text { Rezistenţa la rupere, } \mathrm{N} / \mathrm{mm}^{2}\end{array}$ & 4.7 & 3.8 & 4.5 & 7.2 & 13.4 \\
\hline $\begin{array}{l}\text { Elongation at break, } \% \\
\text { Alungirea la rupere, \% }\end{array}$ & 527 & 560 & 120 & 180 & 213 \\
\hline $\begin{array}{l}\text { Tear strength, } \mathrm{N} / \mathrm{mm} \\
\text { Rezistenţa la sfâşiere, } \mathrm{N} / \mathrm{mm}\end{array}$ & 49 & 34 & 38.5 & 49.5 & 77 \\
\hline
\end{tabular}

\section{Establishing the EB Action onto the Physico- Mechanical Characteristics of Blends}

As noticed from results presented in Table 3, obtained EVA/PVC blends have poor physicomechanical characteristics compared to those of their components, indicating a low degree of miscibility between the two phases of EVA/PVC blends. In order to improve physico-mechanical properties of blends, samples are irradiated with EB. Figures 1-6 present the influence of physico-mechanical characteristics

\section{Stabilirea influenţei EA asupra caracteristicilor fizico- mecanice ale amestecurilor}

Aşa cum se observă din rezultatele prezentate în Tabelul 3, amestecurile EVA/PVC obţinute au caracteristici fizico-mecanice slabe comparativ cu cele ale materialelor componente, indicând un grad scăzut de miscibilitate între cele două faze ale amestecurilor EVA/PVC. Pentru îmbunătăţirea proprietăţilor fizicomecanice ale amestecurilor se realizează iradierea cu EA a probelor. În Figurile 1-6 este prezentată influenţa 
depending on the irradiation dose for EVA/PVC blends with various compositions. The obtained results reveal that upon increasing the irradiation dose, for all blends, there was an increase in hardness, elasticity, $100 \%$ modulus, a decrease in elongation at break, and tensile and tear strength showed a maximum specific for each composition, following a slight decrease. These changes are more prominent in blends with a higher EVA content. They occur as a result of: (1) EVA crosslinking (formation of crosslinking bridges between EVA macromolecules) by EB irradiation, leading to an increase in hardness, elasticity, $100 \%$ modulus, tensile strength and tear strength and a decrease in elongation at break; (2) formation of a copolymer at the interface of the two phases as a result of EB action, which has the role of compatibilizer and leads to improving properties by optimization of interfacial tension, enhanced adhesion between the phases in the solid state etc.

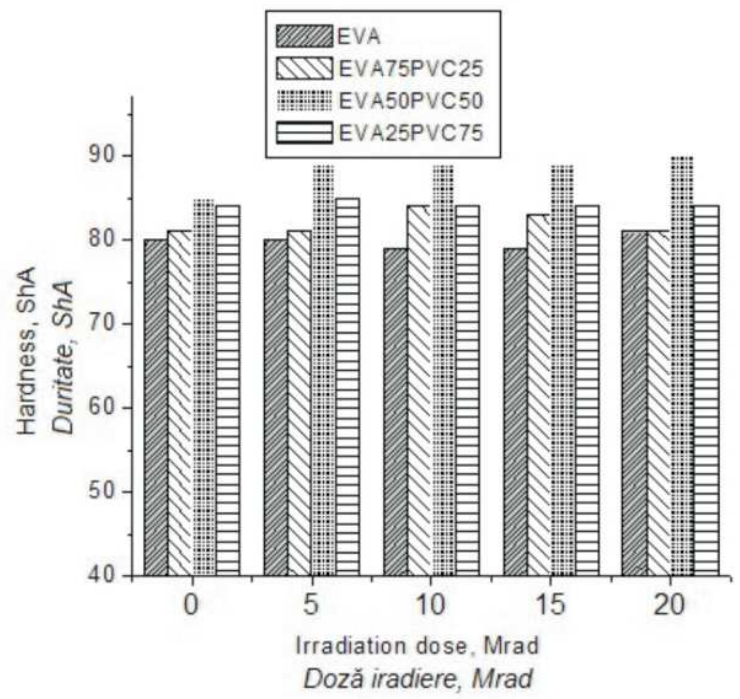

Figure 1. Hardness versus EB irradiation dose and blends type

Figura 1. Duritatea fiecărui tip de amestec în funcţie de doza de iradiere cu EA caracteristicilor fizico-mecanice în funcţie de doza de iradiere pentru amestecurile EVA/PVC cu diferite compoziţii. Rezultatele obţinute arată că la creşterea dozei de iradiere, la toate amestecurile are loc o creştere a durităţii, elasticităţii, modulului 100\%, o scădere a alungirii la rupere, iar rezistenţa la rupere şi rezistenţa la sfâşiere au prezentat o valoare maximă specifică pentru fiecare compoziţie în parte, urmată de o uşoară scădere. Aceste modificări sunt mai accentuate la amestecurile cu un conţinut mai mare de EVA. Ele apar ca urmare a: (1) reticulării EVA (formării unor punţi de reticulare între macromoleculele de EVA) prin iradiere cu EA care conduce la creşterea durităţii, elasticităţii, modulului $100 \%$, rezistenţei la rupere şi rezistenţei la sfâşiere şi la scăderea alungirii la rupere; (2) formării unui copolimer la interfaţa dintre cele două faze sub acţiunea EA, care are rol de compatibilizator şi conduce la îmbunătăţirea proprietăţilor prin optimizarea tensiunii interfaciale, adeziunea îmbunătăţită între faze în starea solidă etc.

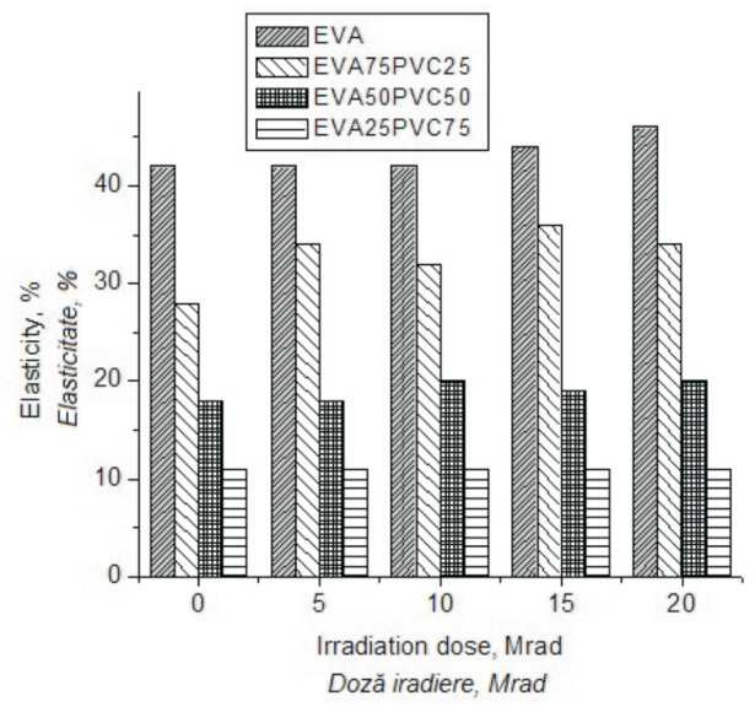

Figure 2. Elasticity versus EB irradiation dose and blends type

Figura 2. Elasticitatea fiecărui tip de amestec în funç̧ie de doza de iradiere cu EA 


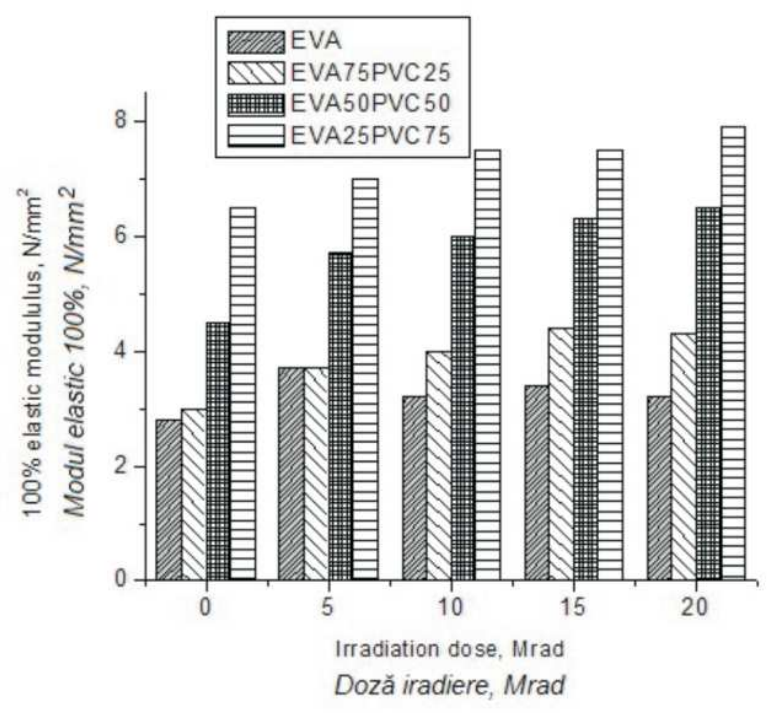

Figure 3. $100 \%$ elastic modulus versus EB irradiation dose and blends type Figura 3. Modulul elastic $100 \%$ al fiecărui tip de amestec în funcţie de doza de iradiere cu EA

\section{Establishing the TAC Amount Action onto the Characteristics of an EB Irradiated EVA/PVC Blend}

Chemical additives known as coagents are used in conjunction with radical cure systems such as organic peroxides and electron beam (EB) irradiation. They are used in the cure of elastomers to promote crosslinking reactions and improve physical properties. Coagents are typically polyfunctional monomers (PFMs) and can participate in a number of radical reaction mechanisms, the most beneficial being grafting and radical addition. By increasing the crosslink density of the compound, network performance can be improved [10-13]. From previous studies [13-14] it was concluded that the most efficient PFM for EVA copolymer blends has been triallylisocyanurate (TAC).

In our experiments, the EVA/PVC blend (25\% EVA and $75 \%$ PVC mass proportion reported to the amount of polymer in the blend) was grafted and crosslinked by means of the accelerated electrons in the presence of a multifunctional monomer - TAC, and the influences of the TAC percentage and accelerated electron irradiation dose on the physical-mechanical characteristics of the samples were investigated. The obtained results reveal that (Figures 7-12): all physico-

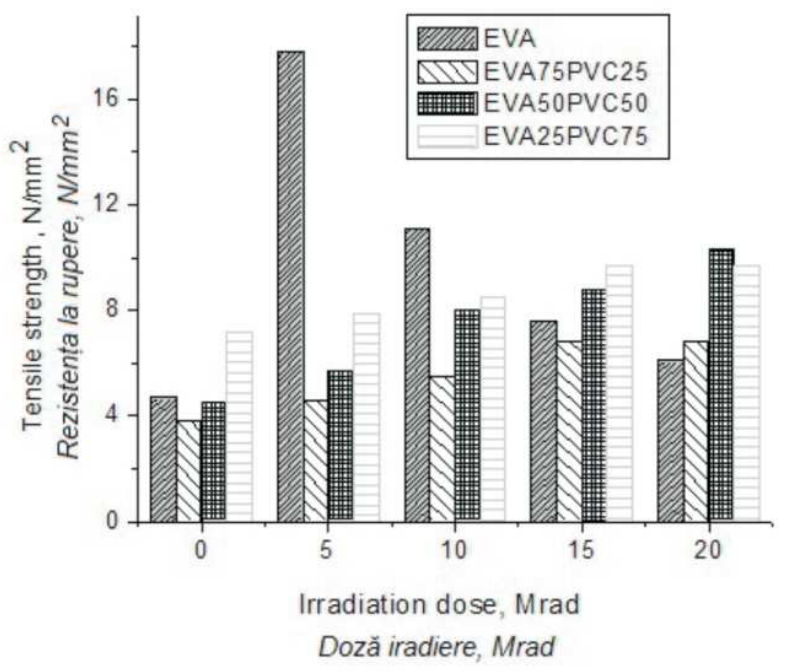

Figure 4. Tensile strength versus EB irradiation dose and blends type Figura 4. Rezistenţa la rupere a fiecărui tip de amestec în funcţie de doza de iradiere cu EA

\section{Stabilirea influenţei cantităţii de TAC asupra caracteristicilor unui amestec EVA/PVC iradiat cu EA}

Aditivii chimici cunoscuţi drept coagenţi sunt utilizaţi în conjunç̧ie cu sisteme de vulcanizare radicală, precum peroxizii organici şi iradierea $\mathrm{cu}$ electroni acceleraţi (EA). Aceştia sunt utilizaţi în vulcanizarea elastomerilor pentru a facilita reacţiile de reticulare şi pentru a îmbunătăţi proprietăţile fizice. Coagenţii sunt de obicei monomeri polifuncţionali (PFMs) şi pot participa într-o serie de mecanisme de reacţii radicale, cele mai avantajoase fiind grefarea şi adiţia radicalică. Performanţa reţelei se poate îmbunătăţi prin creşterea densităţii de reticulare a compusului [10-13]. Din studiile anterioare [13-14] s-a concluzionat că cel mai eficient PFM pentru amestecurile de copolimeri EVA a fost trialilizocianuratul (TAC).

În experimentele noastre, amestecul EVA/PVC (25\% EVA şi 75\% PVC proporţie de masă raportat la cantitatea de polimer din amestec) a fost grefat şi reticulat utilizând electroni acceleraţi în prezenţa unui monomer multifuncţional - TAC şi s-a investigat influenţa cantităţii de TAC şi a dozei de iradiere cu electroni acceleraţi asupra caracteristicilor fizicomecanice ale probelor. Rezultatele obţinute arată că (Figurile 7-12): toate caracteristicile fizico-mecanice se 
mechanical characteristics improve for blends containing TAC (Figures 7-12) compared to similar ones in which TAC was not introduced (Figures 1-6), the highest increases were for $100 \%$ modulus, tensile strength and tear strength. The best results were obtained for blends containing 3 phr TAC and 6 phr TAC, respectively.

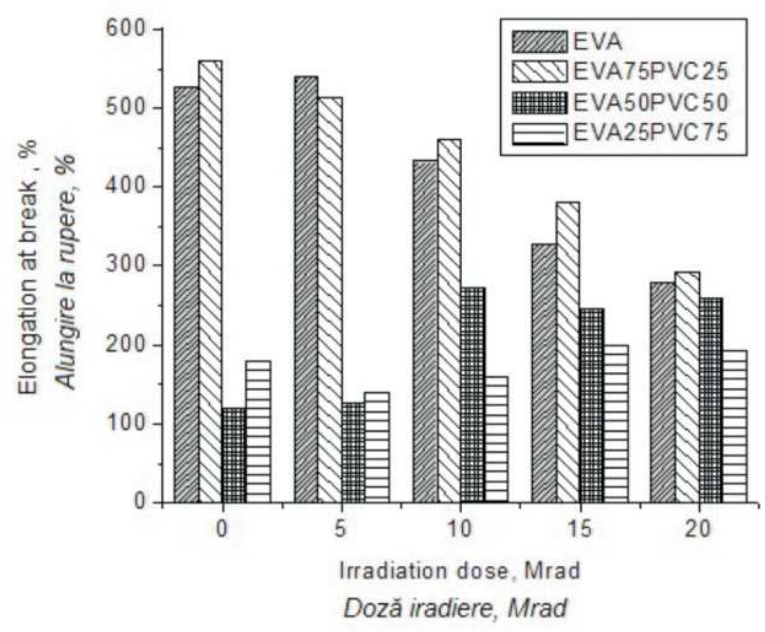

Figure 5. Elongation al break versus $E B$ irradiation dose and blends type Figura 5. Alungirea la rupere a fiecărui tip de amestec în funcţie de doza de iradiere cu EA

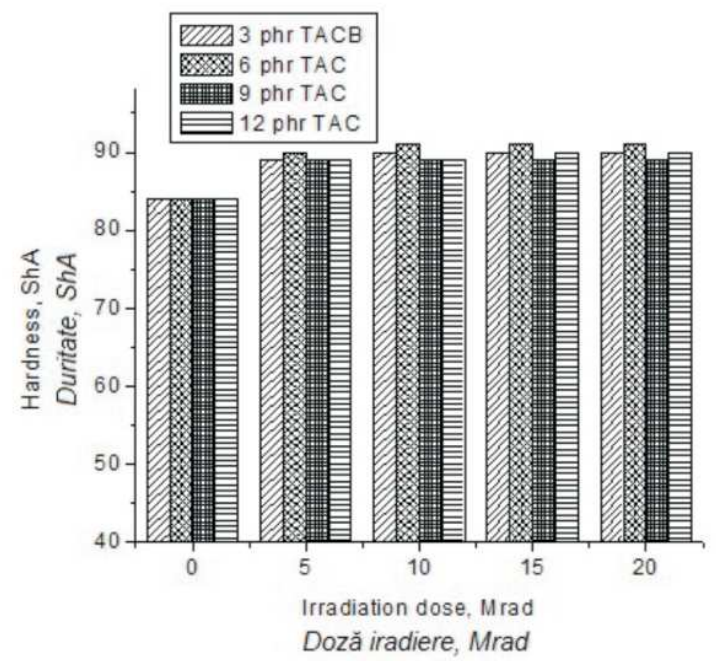

Figure 7. Hardness versus EB irradiation dose and TAC concentration

Figura 7. Duritatea în funcţie de doza de iradiere cu EA şi concentraţia TAC îmbunătățesc la amestecurile care conţin TAC (Figurile 7-12) comparativ cu cele similare în care nu s-a introdus TAC (Figurile 1-6), cele mai mari creşteri au fost pentru modulul $100 \%$, rezistenţa la rupere şi rezistenţa la sfâşiere. La amestecurile care conţin 3 phr TAC, respectiv 6 phr TAC s-au obţinut cele mai bune rezultate.

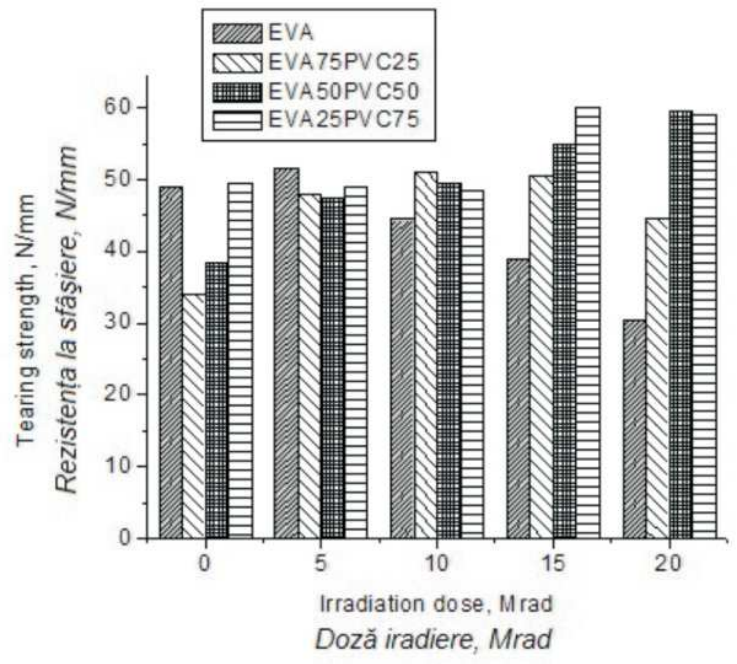

Figure 6. Tearing strength

versus EB irradiation dose and blends type

Figura 6. Rezistenţa la sfâşiere a fiecărui tip de amestec în funcţie de doza de iradiere cu EA

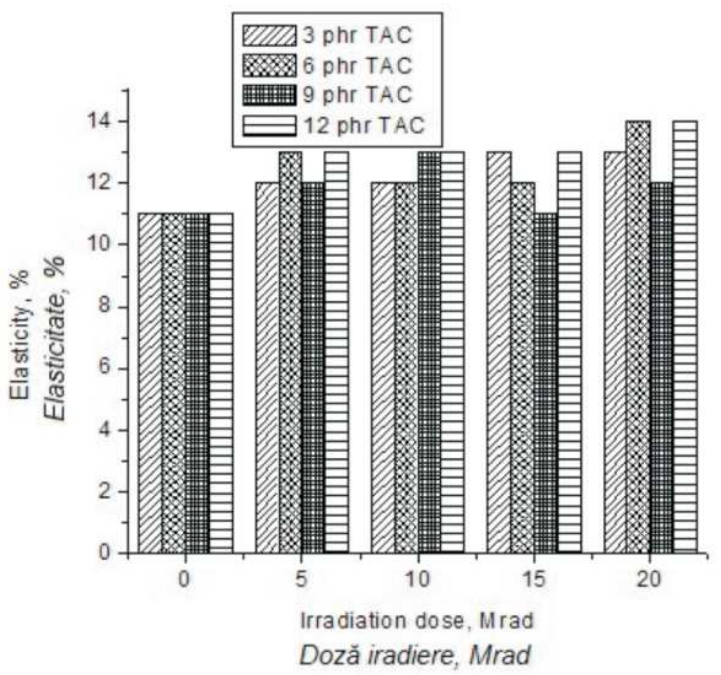

Figure 8. Elasticity versus EB irradiation dose and TAC concentration

Figura 8. Elasticitatea în funcţie de doza de iradiere cu EA şi concentraţia TAC 


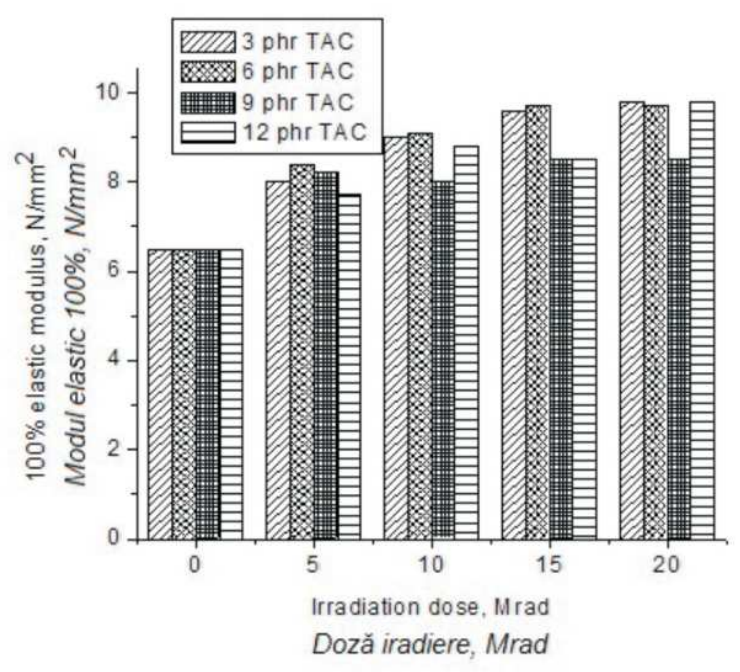

Figure 9. $100 \%$ elastic modulus versus EB irradiation dose and TAC concentration Figura 9. Modulul elastic $100 \%$ în funcţie de doza de iradiere cu EA şi concentraţia TAC

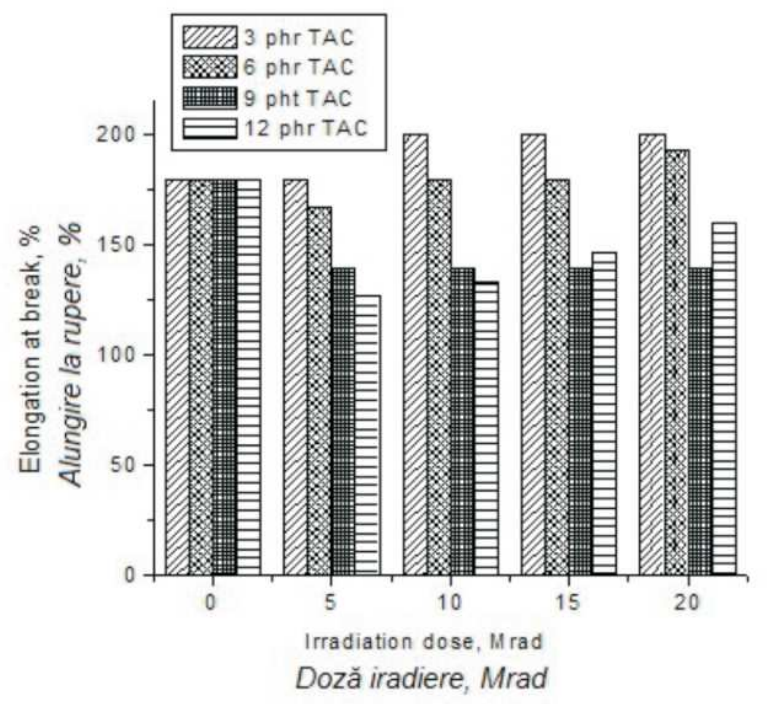

Figure 11. Elongation at break versus EB irradiation dose and TAC concentration Figura 11. Alungirea la rupere în funcţie de doza de iradiere cu EA şi concentraţia TAC

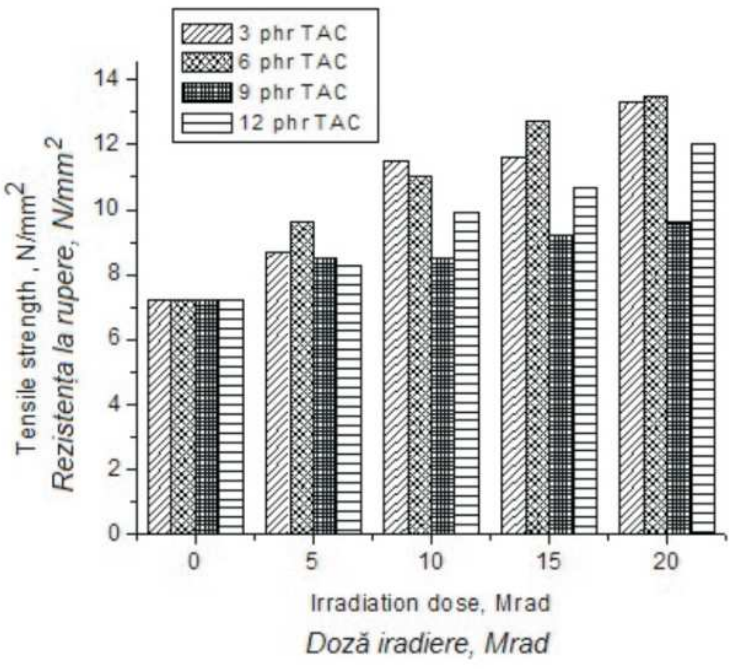

Figure 10. Tensile strength

versus $\mathrm{EB}$ irradiation dose and TAC concentration Figura 10. Rezistenţa la rupere în funcţie de doza de iradiere cu EA şi concentraţia TAC

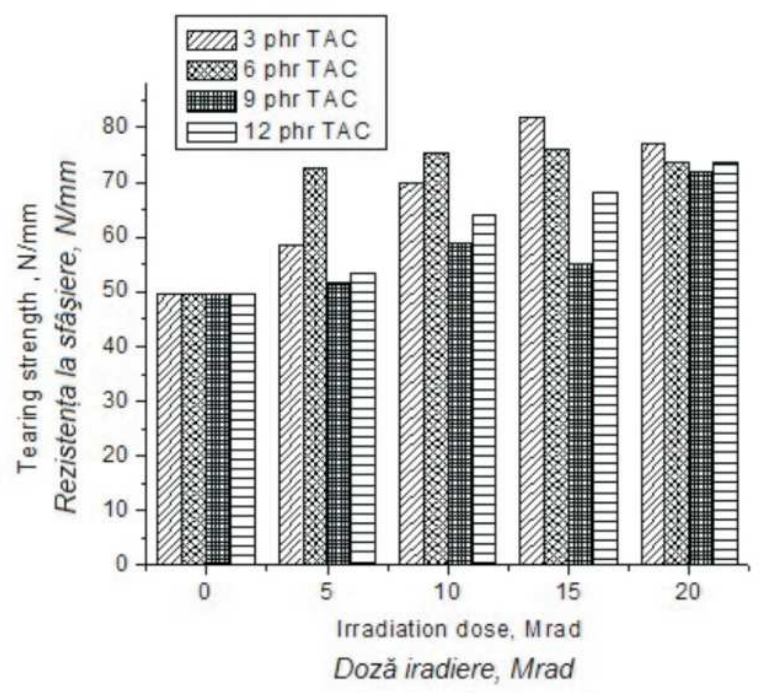

Figure 12. Tearing strength versus EB irradiation dose and TAC concentration Figura 12. Rezistenţa la sfâşiere în funcţie de doza de iradiere cu EA şi concentraţia TAC 


\section{CONCLUSIONS}

By irradiating blends of EVA/PVC in presence of TAC, new materials with improved properties have been obtained, as a result of crosslinking reactions and reorientation of sample particles under the influence of accelerated electrons. The best irradiation dose and the best TAC concentration can be chosen depending on the composition of each blend and on the applications of the final product.

\section{CONCLUZII}

Prin iradierea amestecurilor de EVA/PVC în prezenţa TAC, s-au obţinut noi materiale cu proprietăţi îmbunătăţite, ca urmare a reacţiilor de reticulare şi reorientare a particulelor probelor sub influenţa electronilor acceleraţi. Doza optimă de iradiere şi concentraţia optimă de TAC se pot alege în funcţie de compoziţia fiecărui amestec în parte şi de utilizările produsuluifinal.

\section{REFERENCES}

1. Righettia, M.C., Cardelli, C., Scalari, M., Tombari, E., Conti, G., Thermodynamics of mixing of poly(vinyl chloride) and poly(ethylene-co-vinyl acetate), Polymer, 2002, 43, 5035-5042.

2. Thaumaturgo, C., Monteiro, E.C., Thermal stability and miscibility in PVC/EVA blends, J Thermal Anal, 1997, 49, 1, 247-254.

3. Rellick, G.S., Runt, J., A dielectric study of poly(ethylene-co-vinylacetate)-poly(vinyl chloride) blends. I. Miscibility and phase behavior, J Polym Sci, Polym Phys, 1986, 24, 2, 279-302.

4. Paul, D.R., Barlow, J.W., A binary interaction model for miscibility of copolymers in blends, Polymer, 1984, 25, 4, 487494.

5. Paul, D.R., Barlow, J.W., Keskkula, H., Polymer blends, in: Mark, H.F., Bikales, N.M., Overberger, C.G., Menges, G., eds., Encyclopedia of polymer science and engineering, vol. 12, New York: Wiley Interscience, 1989.

6. Utracki, L.A., Compatiblization of Polymer Blends, Can J Chem Eng, 2002, 80, 1008-1016.

7. Stelescu, M.D., Airinei, A., Grigoras, C., Niculescu-Aron, I.G., Use of Differential Scanning Calorimetry (DSC) in the Characterization of EPDM/PP Blends, Int J Thermophys, 2010, 31, 11-12, 2264-2274.

8. Stelescu, M.D., Manaila, E., New Technology for Obtaining High-Performance Multifunctional Elasto-Plastic Materials, II International Leather Engineering Congress Innovative Aspects for Leather Industry, Izmir, Turkey, May 12-13, 2011, 311-316.

9. Jinhua, W., Yoshii, F., Makuuchi, K., Radiation Vulcanization of ethylene-propylene rubber with polyfunctional monomers, Radiat Phys Chem, 2001, 60, 1-2, 139-142.

10. Stelescu, M.D., Manaila, E., Zuga, N., The use of polyfunctional monomers in the radical cure of chlorinated polyethylene, Polym J, 2011, 43, 9, 792-800.

11. Vijayabaskar, V., Bhowmick, A.K., J. Appl. Polym. Sci., 2005, 95, 2, 435-447.

12. Bhattacharya, A., Prog. Polym. Sci., 2000, 25, 371-401.

13. Dutta, S.K., Bhowmick, A.K., Chaki, T.K., Structure property relationship of ethylene vinyl acetate copolymer grafted with triallyl cyanurate by electron beam radiation, Radiat Phys Chem, 1996, 47, 6, 913-926.

14. Stelescu, M.D., Manaila, E., Craciun, G. et al., Crosslinking and grafting ethylene vinyl acetate copolymer with accelerated electrons in the presence of polyfunctional monomers, Polym Bull, 2012, 68, 1, 263-285.

15. Technical Reports Series No. 277, Absorbed dose determination in photon and electron beams. International Atomic Energy Agency, 4, Vienna, 1987.

16. Holm, N.W., Berry, R.J. (eds.), Manual on radiation dosimetry, Marcel Dekker, New York, 1970. 
17. Lee, D.W., Thermal Degradation Of Polymer Blends Containing Poly(Vinyl Chloride), Thesis submitted in partial fulfillment of the requirements for the degree of master of science, Rochester Institute of Technology, Rochester, New York 14623, Department of Chemistry, 1987. 